\title{
A New Method for Spatial Analysis of Risk in Water Resources Engineering Management
}

\author{
Slobodan P. Simonovic ${ }^{*}$ and Shohan S. Ahmad ${ }^{*}$ \\ Department of Civil and Environmental Engineering, The University of Western Ontario, London, Ontario, Canada, \\ N6A $5 B 9$
}

\begin{abstract}
Uncertainty in water resources management is in part about variability, in part about ambiguity. Both are associated with lack of clarity because of the behavior of all system components, lack of data, lack of detail, lack of structure to consider the water resources management problems, working and framing assumptions being used to consider the problems, known and unknown sources of bias, and ignorance about how much effort it is worth expending to clarify the management situation. The two major sources of variability are temporal and spatial heterogeneity. Temporal variability occurs when values fluctuate with time. Other values which are affected by spatial variability are dependent upon location of an area. A major part of the water resources management risk confusion relates to an inadequate distinction between the objective risk (real, physical) and subjective (perceived) risk. Because of the confusion between the two concepts, many characteristics of subjective risk are believed to be valid also for objective risk. The main objective of this paper is to present the possible methodology for the reliability analysis of water resources systems that will be capable of: (a) addressing water resources uncertainty caused by variability and ambiguity; (b) integrating objective and subjective risk; and (c) assisting the water resources management based on better understanding of spatial variability of risk. Presented methodology is illustrated using flood reliability analysis of the Medway Creek floodplain in the City of London, Ontario, Canada.
\end{abstract}

\section{INTRODUCTION OF RESEARCH CONTEXT}

\section{Water Resources Management Under Uncertainty}

Uncertainty is in the plain language defined as lack of certainty. It has important implications for what can be achieved by water resources systems management. All water management decisions should take uncertainty into account. Sometimes the implications of uncertainty are risk in the sense of significant potential unwelcome effects of water resources system performance. Then managers need to understand the nature of the underlying threats in order to identify, assess and manage the risk. Failure to do so is likely to result in adverse impacts on performance, and in extreme cases, major performance failures. Sometimes the implications of uncertainty are an opposite form of risk, significant potential welcome effects. Then managers need to understand the nature of the underlying opportunities in order to identify and manage the associated decrease in risk. Failure to do so can result in a failure to capture good luck, which can increase the risk. For example, a development of regional water supply system which generates unexpectedly rapid urbanization of the area may prove a disaster if the increasing demand can not be met in the future; a pipeline construction project activity which finishes early may not result in a following activity starting early, and later delays will not be avoided by this good luck if it is wasted; a structural flood protection measure which generates new opportunities for the development of floodplain may increase the future damage in the case of a more severe flood event.

*Address correspondence to these authors at the Department of Civil and Environmental Engineering, The University of Western Ontario, London, Ontario, Canada, N6A 5B9;

E-mail: simonovic@uwo.ca orsahmad52@uwo.ca
Uncertainty is in part about variability in relation to physical characteristics of the water resources systems. But uncertainty is also about ambiguity [1,2]. Both variability and ambiguity are associated with lack of clarity because of the behavior of all system components, lack of data, lack of detail, lack of structure to consider the water resources management problems, working and framing assumptions being used to consider the problems, known and unknown sources of bias, and ignorance about how much effort it is worth expending to clarify the management situation.

\section{Time and Space}

Uncertainty caused by variability is a result of inherent fluctuations in the quantity of interest (hydrologic variables). The three major sources of variability are temporal, spatial and individual heterogeneity. Temporal variability occurs when values fluctuate with time. Other values which are affected by spatial variability are dependent upon location of an area. The third category of individual heterogeneity effectively covers all other sources of variability. In water resources management variability is mainly associated with the spatial and temporal variation of hydrological variables (precipitation, river flow, water quality parameters, etc.).

The more elusive type of uncertainty is ambiguity which is due to a fundamental lack of knowledge. It occurs when the particular values that are of interest cannot be presented with complete confidence because of a lack of understanding or limitation of knowledge.

\section{Risk Definition}

An attempt to come up with a standardized definition of risk, concluded that a common definition is perhaps unachievable and that authors should continue to define risk in their own way. As a result, a numerous definitions can be found in current literature. At a conceptual level, risk is de- 
fined as significant potential unwelcome effect of water resources system performance or the predicted or expected likelihood that a set of circumstances over some time frame will produce some harm that matters. More pragmatic treatments view risk as one side of an equation, where risk is equated with the probability of failure or the probability of load exceeding resistance. Other symbolic expressions equate risk with the sum of uncertainty and damage or the quotient of hazards divided by safeguards [3].

Three cautions surrounding risk must be taken into consideration: risk cannot be represented objectively by a single number alone, risks cannot be ranked on strictly objective grounds, and risk should not be labelled as real. Regarding the caution of viewing risk as a single number, the multidimensional character of risk can only be aggregated into a single number by assigning implicit or explicit weighting factors to various numerical measures of risk. Since these weighting factors must rely on value judgement, the resulting single metric for risk can not be objective. Since risk can't objectively be expressed by a single number, it is not possible to rank risks on strictly objective grounds. Finally, since risk estimates are evidence-based, risks can't be strictly labelled as real. Rather, they should be labelled inferred at best.

\section{Objective and Subjective Risk}

A major part of the risk management confusion relates to an inadequate distinction between three fundamental types of risk: (i) Objective risk (real, physical), $\mathrm{R}_{0}$, and objective probability, $\mathrm{p}_{\mathrm{o}}$, which is the property of real physical systems.; (ii) Subjective risk, $\mathrm{R}_{\mathrm{s}}$, and subjective probability, $\mathrm{p}_{\mathrm{s}}$. Probability is here defined as the degree of belief in a statement. $R_{s}$ and $p_{s}$ are not properties of the physical systems under consideration (but may be some function of $R_{0}$ and $\mathrm{p}_{\mathrm{o}}$ ); and (iii) Perceived risk, $\mathrm{R}_{\mathrm{p}}$, which is related to an individual's feeling of fear in the face of an undesirable possible event, is not a property of the physical systems but is related to fear of unknown. It may be a function of $R_{o}, p_{o}, R_{s}$, and $p_{s}$. Because of the confusion between the concepts of objective and subjective risk, many characteristics of subjective risk are believed to be valid also for objective risk [4]. Therefore, it is almost universally assumed that the imprecision of human judgment is equally prominent and destructive for all water resources risk evaluations and all risk assessments. This is perhaps the most important misconception that blocks the way toward more effective societal risk management. The ways society manages risks appear to be dominated by considerations of perceived and subjective risks, while it is objective risks that kill people, damage the environment and create property loss.

The main objective of this paper is to present one of the possible methodology for the reliability analysis of water resources systems that will be capable of: (a) addressing water resources uncertainty caused by variability and ambiguity; (b) integrating objective and subjective risk; and (c) assisting the water resources management based on better understanding of temporal and spatial variability of risk.

The following section provides an overview of previous work. The introduction of state-of-the-art methodology based on the spatial fuzzy reliability analysis follows. An illustration of the application of presented methodology to flood reliability analysis ends the paper.

\section{PREVIOUS WORK}

\section{Probabilistic Approach}

Probability is a concept widely accepted and practiced in water resources systems management. To perform operations associated with probability, it is necessary to use sets - collection of elements, each with some specific characteristics.

In the classical interpretation of probability (Equally Likely Concept, the probability of an event $E$ can be obtained from $\operatorname{Pr}(E)=m(E) / m(S)$, provided that the sample space contains $N$ equally likely and different outcomes, i.e., $m(S)=N, n$ of which have an outcome (event) $E$, i.e., $m(E)$ $=n$. Thus $\operatorname{Pr}(E)=n / N$. This definition is often inadequate for water resources applications. For example, if failures of a pump to start in a water supply plant are observed, it is unknown whether all failures are equally likely to occur. Nor is it clear if the whole spectrum of possible events is observed.

In the frequency interpretation of probability, the limitation on the lack of knowledge about the overall sample space is remedied by defining the probability as the limit of $n / N$ as $N$ becomes large. Therefore, $\operatorname{Pr}(E)=\lim _{\mathrm{N} \rightarrow \infty}(n / N)$. Thus if we have observed 2000 starts of a pump in which 20 failed, and if we assume that 2000 is a large number, then the probability of the pump failure to start is $20 / 2000=0.01$. The frequency interpretation is the most widely used classical definition in water resources management today.

\section{Problems with Probabilistic Approach}

The probabilistic (stochastic) reliability analysis has been extensively used to deal with the problem of uncertainty in water resources systems management. Prior knowledge of the probability density functions of both resistance and load and/or their joint probability distribution function is a prerequisite to the probabilistic approach. In practice, data on previous failure experience is usually insufficient to provide such information. Even if data is available to estimate these distributions, approximations are almost always necessary to calculate system reliability [5]. The subjective judgment of the water resources decision maker to estimate the probability distribution of a random event - subjective probability approach of Vick [6] - is another approach to deal with data insufficiency. The third approach is Bayes's theory where engineering judgment is integrated with observed information.

Until recently the probabilistic approach was the only approach for water resource systems reliability analyses. However, it fails to address the problem of uncertainty that goes along with human input, subjectivity, lack of history and records. There is a real need to convert to new approaches that can compensate for the ambiguity or uncertainty of human perception.

\section{Fuzzy Set Approach}

Fuzzy set theory was intentionally developed to try to capture people judgmental believes, or the uncertainty that is caused by the lack of knowledge. Relative to the probability theory, it has some degree of freedom with respect to aggregation operators, types of fuzzy sets (membership functions), 
etc, which enables the adaptability to different contexts. Probability and fuzziness are related, but different concepts. Fuzziness is a type of deterministic uncertainty. It describes the event class ambiguity. Fuzziness measures the degree to which an event occurs, not whether it occurs. At issue is whether the event class can be unambiguously distinguished from its opposite. Probability arouses from the question whether or not an event occurs. Moreover, it assumes that the event class is crisply defined and that the law of non contradiction - for any property and for any definite subject, it is not the case both that the subject possesses that property and that the subject does not possess that property - holds. Fuzziness occurs when the law of non contradiction (and equivalently the law of excluded middle - for any property and for any individual, either that individual possesses that property or that individual does not possess that property) is violated. However, it seems more appropriate to investigate the fuzzy probability for the latter case, than to completely dismiss probability as a special case of fuzziness.

In essence, whenever there is an experiment for which we are not capable of "computing" the outcome, a probabilistic approach may be used to estimate the likelihood of a possible outcome belonging to an event class. A fuzzy theory extends the traditional notion of a probability when there are the outcomes that belong to several event classes at the same time but to different degrees. The fuzziness and probability are orthogonal concepts that characterize different aspects of human experience. Hence, it is important to note that neither fuzziness nor probability govern the physical processes in Nature. They are introduced by humans to compensate for our own limitations.

\section{A NEW METHODOLOGY FOR SPATIAL ANALY- SIS OF RISK IN WATER RESOURCES MANAGE- MENT}

\section{Fuzzy Risk Definition and Analysis}

A new methodology starts with a definition of partial failure that provides for the water resource systems reliability analysis using three fuzzy performance measures: (i) a combined reliability-vulnerability index, (ii) a robustness index, and (iii) a resiliency index [7]. The calculation of performance indices depends on the exact definition of unsatisfactory system performance. Water resources systems reliability analysis uses load and resistance to define the state of a system. The failure state occurs when resistance falls below the load. It is difficult to arrive at a precise definition of failure because of the uncertainty in determining system resistance, load, and the accepted unsatisfactory performance threshold. Fig. (1) depicts a typical system performance (resistance time series), with a constant load during the operation horizon. According to the classical definition, the failure state is the state when resistance falls below the load, margin of safety (difference between the resistance and load) $\mathrm{M}<0.0$ or safety factor $\Theta<1.0$, which is represented by the ratio between the system's resistance and load, shown in Fig. (1) by the dashed horizontal line.

Due to the fluctuation of load and resistance in the management of water resources systems, partial failure may be acceptable. The precise identification of failure is neither realistic nor practical. A degree of acceptable system failure is introduced using the solid horizontal line, as shown in Fig. (1). The region between the dashed and the full line in the figure is the region of partial failure that will be called acceptable failure.

The boundary of the acceptable or partial failure region is ambiguous and varies from one decision maker to the other depending on the personal perception of risk. Fuzzy sets are capable of representing the notion of imprecision better than ordinary sets and therefore, the acceptable level of performance can be represented as a fuzzy membership function shown in Fig. (2).

The reliability assessment, discussed here, involves a comparative analysis of the system-state membership function (see Fig. 2) and the predefined acceptable level of the performance membership function. Therefore, the compliance of two fuzzy membership functions can be quantified using the fuzzy compatibility measure. Possibility and necessity lead to the quantification of the compatibility of two fuzzy sets. The possibility measure quantifies the overlap between two fuzzy sets, while the necessity measure de-

Fig. (1). Variable system performance. 
Fig. (2). Fuzzy representation of the acceptable level of performance and system state.

scribes the degree of inclusion of one fuzzy set into another fuzzy set.

Analysis of the overlap between two fuzzy membership functions provides for definition of reliability and vulnerability as a complete descriptor of system performance. Once an acceptable level of performance is determined in a fuzzy form, the anticipated performance in the event of failure as well as the expected severity of failure can be determined. A comparison between the fuzzy system-state membership function and the predefined fuzzy acceptable level of performance membership function provides information about both, system reliability and system vulnerability at the same time.

Robustness measures the system's ability to adapt to a wide range of possible future load conditions. The fuzzy form of change in future conditions can be obtained through a redefinition of the acceptable level of performance and a change in the system-state membership function. As a result, the system's robustness is defined as the change in the compatibility measure - overlap of two fuzzy membership functions.

Resilience measures how fast the system recovers from failure state. The time required to recover from the failure state can be represented as a fuzzy set. A series of fuzzy membership functions can be developed to allow for various types of failure. The maximum recovery time is used to represent the system recovery time. The center of gravity of the maximum fuzzy recovery time can be used as a real number representation of the system recovery time. Therefore, system resilience is determined to be the inverse value of the center of gravity. Fuzzy reliability analysis has been successfully tested on the City of London (Ontario, Canada) Regional Water Supply System [8].

\section{Spatial Fuzzy Reliability Analysis}

Integration of fuzzy performance indices computation with GIS allows for spatial fuzzy reliability analysis. Each cell in a GIS map is considered a decision making location for which the computation of fuzzy indices is done as described in the previous section [9].

\section{FUZZY FLOOD RELIABILITY ANALYSIS - A CASE STUDY}

The utility of the methodology has been tested in reliability analysis of floods. The fuzzy flood damage membership functions for agricultural land, residential land, one and two story buildings are developed based on the flood damage data. The compliance of the flood damage membership function with different acceptable levels of flood damage is assessed for every grid cell. The maximum value of the compatibility measures for every grid cell in space is combined into a single raster image. The designated maps are used to determine the fuzzy combined reliability-vulnerability, fuzzy robustness and fuzzy resiliency indices. The spatial representation of the fuzzy reliability indices provides for easier identification of flood prone areas and better understanding of spatial variation of flood risk.

\section{Fuzzy Flood Damage in Space}

In this research, a triangular membership function is used to represent the flood damage. Every grid cell in GIS contains flood damage defined by the membership function $\tilde{S}(D)$ on the universe of discourse D:

$$
\tilde{S}(D)=\left\{\begin{array}{cc}
0 & \text { if } D \leq D_{\text {Min }} \\
\frac{D-D_{\text {Min }}}{D_{\text {Mean }}-D_{\text {Min }}} & \text { if } D \in\left[D_{\text {Min }}, D_{\text {Mean }}\right] \\
\frac{D_{\text {Max }}-D}{D_{\text {Max }}-D_{\text {Mean }}} & \text { if } D \in\left[D_{\text {Mean }}, D_{\text {Max }}\right] \\
0 & \text { if } D \geq D_{\text {Max }}
\end{array}\right\}
$$

where, $\tilde{S}(D)$ is the flood damage membership function; $D_{\text {Mean }}$ is the modal value of the flood damage; and $D_{\text {Min }}, D_{M a x}$ are the lower and the upper bounds of the flood damage value.

\section{Fuzzy Acceptable Level of Flood Damage in Space}

The fuzzy acceptable level of flood damage is set for the raster image. It is represented as a fuzzy membership function, $\tilde{M}(D)$ : 


$$
\tilde{M}(D)=\left\{\begin{array}{cc}
1 & \text { if } D \leq D_{1} \\
\theta(D) & \text { if } D \in\left[D_{1}, D_{2}\right] \\
0 & \text { if } D \geq D_{2}
\end{array}\right\}
$$

where, $\tilde{M}(D)$ is the fuzzy membership function of margin of safety; and $D_{l}, D 2$ are the lower and the upper bounds of the acceptable level of flood damage.

If the value of flood damage exceeds $\mathrm{D}_{2}$, then the region suffers complete damage (Fig. 3). In this case the membership function $\tilde{M}(D)$ value is zero. If the value of flood damage is below $\mathrm{D}_{2}$ but exceeds $\mathrm{D}_{1}$, then the region suffers partial flood damage. The membership function, $\tilde{M}(D)$ of the acceptable level of flood damage attains its maximum value of one if the value of flood damage is below $\mathrm{D}_{1}$.

Process of calculating fuzzy performance indices is based on the use of two input membership functions, as defined by equation (1) and (2). Mathematical transformations of information from the input membership functions involve calculation of fuzzy reliability and fuzzy compatibility.

Fuzzy reliability (LR) of the acceptable level of flood damage is calculated for every grid cell in the raster image as follows:

$$
L R=\frac{D_{1} \times D_{2}}{D_{2}-D_{1}}
$$

Weighted area of the flood damage membership function and the weighted overlap area between the acceptable level of flood damage membership function and the flood damage membership function (Fig. 3) are calculated in GIS for determining the level of compliance.

Fuzzy compatibility (CM) between the fuzzy flood damage membership function and the fuzzy acceptable level of flood damage membership function is the basis for the calculation of the fuzzy combined reliability-vulnerability index.
It is expressed as follows [7]:

Compatibility Measure (CM)

$$
=
$$

Weighted overlap area

Weighted area of system - state function

\section{Spatial Fuzzy Combined Reliability-Vulnerability Index}

Fuzzy reliability and fuzzy compatibility of two input membership functions are used in mathematical derivation of the combined fuzzy reliability-vulnerability index which is calculated for each grid cell in GIS:

$$
R E_{i}=\frac{\max _{i \in K}\left\{C M_{1}, C M_{2}, \ldots \ldots . . C M_{i}\right\} \times L R_{\max }}{\max _{i \in K}\left\{L R_{1}, L R_{2} \ldots \ldots . . L R_{i}\right\}} \quad \ldots
$$

where, $R E_{i}$ is the combined fuzzy reliability-vulnerability index for grid cell $\mathrm{i} ; L R_{\max }$ is the fuzzy reliability of acceptable level of damage corresponding to the flood damage with maximum compatibility value; $L R_{i}$ is the fuzzy reliability of the i-th acceptable level of flood damage; $C M_{i}$ is the fuzzy compatibility for flood damage with the i-th acceptable level of flood damage; and $K$ is the total number of the predefined acceptable levels of flood damage.

In this work three different acceptable levels of flood damage (i.e. conservative, neutral and risky) are considered for lands and buildings. Arbitrary shapes of membership functions corresponding to three different acceptable levels of flood damage are used to capture the range of decision maker's risk perceptions - from risky to conservative (Fig. 4). The compliance of the flood damage with the three acceptable levels of flood damage is obtained from the fuzzy compatibility using the weighted overlap area approach. Then the maximum value of the fuzzy compatibility is considered. Three fuzzy reliability values (LR) are calculated

Fig. (3). Acceptable level of flood damage. 
from three acceptable levels of flood damage and the maximum value of LR is determined and assigned to the designated map [9].

The maximum fuzzy compatibility, the fuzzy reliability of acceptable level of flood damage corresponding to the flood damage with maximum compatibility, and the maximum of the fuzzy reliability are used in Equation (5).

\section{Spatial Fuzzy Robustness Index}

The adaptability of the system to the change in the acceptable level of flood damage is spatially represented in GIS. Two maps containing compatibility measure values are used as inputs in the following equation:

$$
R O_{i}=\frac{1}{C M_{1}-C M_{2}}
$$

where, $R O$ is the fuzzy robustness index for the grid cell $\mathrm{i}$; $C M_{1}$ is the compatibility value before the change in the acceptable level of flood damage; and $\mathrm{CM}_{2}$ is the compatibility value after the change in the acceptable level of flood damage.

The inverse of the difference in compatibility values between the two acceptable levels of flood damage is calculated in each cell of the GIS to give the spatial fuzzy robustness index. The spatial fuzzy robustness index is developed for three cases (Fig. 4): change of the acceptable level of flood damage from: (i) conservative to neutral; (ii) neutral to risky; and (iii) conservative to risky.

\section{Spatial Fuzzy Resiliency Index}

Resiliency index measures the ability of system to recover from the failure state. A resilient community is able to recover quickly from a flood disaster. After a disaster, post flood recovery involves restoring all systems to normal or near normal condition. As a measure of the ability to recover we use the time necessary to recover from flood which de- pends upon factors such as water drainage, damage assessment, provision of flood assistance to flood victims, time for rebuilding or repairing and return to normal life $[10,11]$.

The extent of flood damage to structures as well as agricultural and residential lands is a key factor in assessing the time required to recover from the flood damage. If a structure remains submerged for several hours during the flood, the damage may be extensive. In such case, to prevent the possible high damage in the future, rebuilding the whole structure instead of repairing makes more sense. This decision will increase the cost of recovery as well as the necessary time for recovery. In most cases, high recovery cost corresponds to longer recovery time and vice-versa. Based upon this assumption, a recovery time $v s$ flood damage relationship is generated in this research for assessing the recovery time in the post flood stage. Both, flood damage and damage $v s$ recovery time relationship, are subjected to uncertainty due to lack of data, subjectivity and ambiguity. Therefore, the recovery time is also subject to uncertainty which is represented using a suitable fuzzy membership function. The minimum, modal and maximum flood damage values $\left(\mathrm{D}_{1}\right.$, $\mathrm{D}_{2}$ and $\mathrm{D}_{3}$ ) are required to calculate the minimum, modal and maximum values of the recovery time $\left(\mathrm{t}_{1}, \mathrm{t}_{2}\right.$ and $\left.\mathrm{t}_{3}\right)$ using the recovery time $v s$ flood damage relationship:

$$
\tilde{S}(t)=\left\{\begin{array}{cc}
0 & \text { if } t \leq t_{1} \\
\frac{t-t_{1}}{t_{2}-t_{1}} & \text { if } t \in\left[t_{1}, t_{2}\right] \\
\frac{t_{3}-t}{t_{3}-t_{2}} & \text { if } t \in\left[t_{2}, t_{3}\right] \\
0 & \text { if } t \geq t_{3}
\end{array}\right\}
$$

where, $\tilde{S}(t)$ is the membership function of the flood recovery time; $t_{2}$ is the modal value of the flood recovery time; and $t_{1}$, $t_{3}$ are the lower and the upper bound of the flood recovery time.

Fig. (4). Fuzzy membership function of the recovery time. 
Flood recovery time varies with the extent of the flood damage. The maximum value of the fuzzy flood recovery time is used to represent the flood recovery time. The center of gravity of the fuzzy flood recovery time is calculated for each cell in space as follows:

$C G_{i}=\frac{\int_{t_{1}}^{t_{3}} t \tilde{T}(t) d t}{\int_{t_{1}}^{t_{3}} \tilde{T}(t) d t}$

where, $C G_{i}$ is the center of gravity of the recovery time membership of the $\mathrm{i}$-th cell; $i$ is the subscript of grid cells, and $\mathrm{i}=1,2,3,4, \ldots \ldots \ldots . \mathrm{N} ; N$ is the total number of grid cells; and $\tilde{T}(t)$ is the fuzzy recovery time.

The inverse of the center of gravity of the recovery time is used to spatially represent the resiliency in a single map. The spatial fuzzy resiliency index is calculated as:

$$
R S_{i}=\left[C G_{i}\right]^{-1}=\left[\frac{\int_{t_{1}}^{t_{3}} t \tilde{T}(t) d t}{\int_{t_{1}}^{t_{3}} \tilde{T}(t) d t}\right]^{-1}
$$

where, $R S_{i}$ is the fuzzy resiliency index for the $\mathrm{i}$-th grid cell.

\section{Medway Creek Flooding Analysis}

The Medway Creek, within the City of London, Ontario, Canada is chosen as a study area to illustrate the application of spatial fuzzy reliability analysis to flood management. Fuzzy flood reliability analysis of Medway Creek is carried out from Arva to the confluence of Medway Creek with the North Thames River. London is one of the cities flooded by the Thames River. An extensive system of dikes and three reservoirs have been constructed to provide protection against major floods. The Medway Creek near London has a mean annual flow of $2.6 \mathrm{~m}^{3} / \mathrm{sec}$ which contributes about $7 \%$ to the flow of the Thames River downstream of London. London is experiencing rapid urban development. Forest and agricultural lands are disappearing due to intensive urbanization. The population of the City of London has increased approximately $43 \%$ over the last thirty years (1971 to 2001) and shows similar migration trend as that of greater Toronto. It is expected that the population of London may increase by a total of $67 \%$ by the year 2026 [12]. Increase in urban development causes (a) reduction in woodlands and agricultural lands; (b) increase of the impervious area; and (c) increase in excess runoff; that makes the city more vulnerable to floods.

\section{Data}

The watershed layer is created using Digital Elevation Model (DEM) with respect to the UTM projected coordinate system for zone $17 \mathrm{~N}$ created in year 2002. The DEM of the Upper Thames River Watershed provides the elevation of the ground surface. A feature image containing the type of land use for the whole Upper Thames Watershed is also used for the study region. Twenty four different types of land use are present in the Middlesex county landuse map. The orthoimage of the City of London (photography scale of 1:10,000) from April 2004 is also used together with the polygon features containing shape files of roads and building structures. The location of the building features and road features matched exactly with the ortho-imagery and DEM for the area of interest in the City London. The hydraulic data of the Medway Creek for the study region is acquired from the Upper Thames River Conservation Authority (UTRCA) for flood plain simulation. The hydraulic data include flow values for 2 year, 5 year, 10 year, 25 year, 50 year, 100 year and 500 year return periods at specific cross-sections of the Medway Creek. Flood damage assessment is carried out considering depth-damage relationship for one and two story buildings, residential, and agricultural land. The stagedamage curve for one and two story buildings with basement in the Medway Creek floodplain is developed using the Flood Damage Estimation Guide [13].

\section{Analyses}

The spatial fuzzy flood reliability analysis of Medway Creek is done in GIS using equations (4), (5), (6) and (9). The fuzzy flood damage membership functions for agricultural land, residential land, one and two story buildings are developed based on the flood damage data. The compliance of the flood damage membership function with different acceptable levels of flood damage is assessed for every grid cell in space. The maximum value of the compatibility for every grid cell is combined into a single raster image. The designated maps are used to determine fuzzy combined reliability-vulnerability, fuzzy robustness and fuzzy resiliency indices. The map of combined fuzzy flood reliabilityvulnerability index of the Medway Creek is in Fig. (5).

The GIS maps containing the inverse of the difference in compatibility values between two acceptable levels of flood damage represent the spatial fuzzy robustness index. The fuzzy robustness index measures the adaptability to change in the acceptable level of flood damage. Fig. (6) shows the maps of fuzzy flood robustness index of the Medway Creek for (a) change from conservative to neutral level of the acceptable flood damage and (b) change from conservative to risky level of the acceptable flood damage.

Time to recover from flood damage is determined using recovery time-damage relationship. Uncertainty in the value of recovery time is accounted for using a triangular fuzzy membership function. The map of fuzzy flood resiliency index of the Medway Creek is shown in Fig. (7).

\section{Results and Discussion}

Detailed flood reliability analysis is possible using information from the maps shown in Figs. (5-7). The quantitative analysis of maps of the entire study region containing compatibility values for conservative, neutral and risky level of flood damage are provided in Table 1. Change from one acceptable level to another results in change of the overlapping area that is used to measure compatibility. About 12.22 $\mathrm{km}^{2}$ of the Medway Creek region belongs to low compatibility value (zero) for the conservative level of the acceptable flood damage (Table 1). In the case of neutral acceptable level of the flood damage, area of $9.97 \mathrm{~km}^{2}$ shows zero compatibility. In the case of risky acceptable level of the flood damage, area of $3.32 \mathrm{~km}^{2}$ shows zero compatibility. At higher level of compatibility (for example value of one) areas of Medway Creek corresponding to conservative, neutral and risky levels of the acceptable flood damage are 8.91 $\mathrm{km}^{2}, 14.07 \mathrm{~km}^{2}$ and $23.42 \mathrm{~km}^{2}$, respectively. As expected, an increase in the acceptable level of flood damage results in 
Fig. (5). Combined fuzzy flood reliability-vulnerability index map of the Medway Creek.

larger area of the study region with higher compatibility value.
The increase in acceptable level of the flood damage results in the lower percentage of the Medway Creek area having low compatibility decreases and high percentage of the 
Fig. (6). Fuzzy flood robustness index map of the Medway Creek for change (a) from conservative to neutral level of the acceptable flood damage; (b) from conservative to risky level of the acceptable flood damage.

Medway Creek area having high compatibility increases. For example, at 0.9 compatibility value (Table $\mathbf{1}$ ) increase in area is about $4 \%$ for the switch from conservative to neutral level of the acceptable flood damage, and $74 \%$ for the switch from conservative to risky level of the acceptable flood damage.

Quantitative analysis of the maps for different compatibility values show that the largest area with maximum compatibility is achieved for the risky acceptable level of the flood damage. Therefore the compatibility value map for the risky level of acceptable flood damage is used in the development of the of spatial combined fuzzy flood reliabilityvulnerability index map of the Medway Creek in Fig. (5). The fuzzy flood reliability index is shown in Fig. (5) in the range from zero to one. The maximum value that the fuzzy reliability index can attain is one. If the actual system has a reliability index of one then the system is considered safe or highly reliable with low vulnerability. If the value of fuzzy reliability index is zero, then the system is considered unsafe with low reliability and high vulnerability. In this study a color ramp is used (Fig. 5) to show the combined fuzzy flood reliability-vulnerability index of the Medway Creek. Blue color is used to show low value of the combined fuzzy flood reliability-vulnerability index and the high value is shown in dark brown color.

The combined fuzzy flood reliability-vulnerability index for a region or a location of particular interest can be easily identified using the color ramp. Land close to Medway Creek is found to have low level of flood reliability and high vulnerability. The combined fuzzy flood reliabilityvulnerability index value ranges from 0 (dark blue) to 0.3 (light blue). Yellow marks the areas of higher reliability and lower vulnerability compared to the regions closer to the river. Value of the combined fuzzy flood reliabilityvulnerability index in this region is between 0.31 (light yellow) and 0.45 (dark yellow). Transition to regions with high reliability is indicated by the value of combined index in the range of 0.46 (dark orange) to 0.6 (light orange). Green marks the regions that are more reliable and less vulnerable to floods and the index value is between 0.61 and 0.89 . Regions with highest reliability are shown in brown color with the index value between 0.9 (light brown) and 1.0 (dark brown). The region close to the confluence of Medway Creek and the North Thames is the most prone to flooding. Areas such as these show a very low value of the combined fuzzy reliability-vulnerability index of 0 to 0.3 .

Comparison of the Medway Creek DEM with the map shows, as expected, that the regions of low ground surface elevation are at higher risk from flooding and therefore have a very low combined fuzzy reliability-vulnerability index. The residential area near the confluence may suffer significant flood damage and large area in the south-east part of the map shows low reliability and high vulnerability. Approximately $6.77 \mathrm{~km}^{2}$ of the southern part of the study region near the river ( $43 \%$ of the City of London on the map) is the area of very low reliability (index value from 0 to 0.3 ). Western part of the study region, $6.14 \mathrm{~km}^{2}$ (39\% of the City of Lon- 
Fig. (7). Fuzzy flood resiliency index map of the Medway Creek.

don on the map) is the area of high reliability (index value from 0.9 to 1$)$.

Areas north of the City (northern part of the study region) are mainly agricultural. It is found that the combined fuzzy flood reliability-vulnerability increases in the northern direction. This is contributed to the fact that the flood damage to agricultural land is lower than the flood damage to residential land. Almost $98 \%$ of the agricultural land under continuous row crop and corn shows the index value between 0.7 and 1.0. Almost $99 \%$ of the land under hay has the index value between 0.9 and 1.0. The change in the combined fuzzy flood reliability-vulnerability index is clearly seen on the map - many regions within the urban part of the City of London are in blue (low reliability and high vulnerability); north of the City, the agricultural regions show a transition from blue to green and brown (higher reliability and lower vulnerability). 
Table 1. Area and Percent Change in the Area of the Study Region Corresponding to Different Compatibility Value and Different Level of the Acceptable Flood Damage

\begin{tabular}{|c|c|c|c|c|c|c|}
\hline \multirow{2}{*}{ 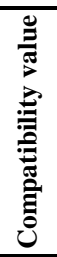 } & \multicolumn{3}{|c|}{$\begin{array}{l}\text { Area }\left(\mathrm{km}^{2}\right) \text { corresponding to different level of the } \\
\text { acceptable flood damage }\end{array}$} & \multicolumn{3}{|c|}{$\begin{array}{l}\text { Percent change in area for changing levels of the } \\
\text { acceptable flood damage }\end{array}$} \\
\hline & Conservative & Neutral & Risky & Conservative to neutral & Neutral to risky & Conservative to risky \\
\hline 0.0 & 12.22 & 9.97 & 3.32 & -18 & -67 & -73 \\
\hline 0.1 & 6.57 & 1.71 & 2.65 & -74 & 55 & -60 \\
\hline 0.2 & 4.51 & 1.34 & 1.53 & -70 & 14 & -66 \\
\hline 0.3 & 3.88 & 4.95 & 1.54 & 28 & -69 & -60 \\
\hline 0.4 & 2.90 & 3.81 & 1.05 & 32 & -72 & -64 \\
\hline 0.5 & 2.77 & 4.14 & 1.12 & 50 & -73 & -60 \\
\hline 0.6 & 3.28 & 3.12 & 1.24 & -5 & -60 & -62 \\
\hline 0.7 & 2.92 & 3.16 & 5.06 & 8 & 60 & 73 \\
\hline 0.8 & 2.55 & 4.07 & 6.75 & 59 & 66 & 165 \\
\hline 0.9 & 3.81 & 3.97 & 6.63 & 4 & 67 & 74 \\
\hline 1.0 & 8.91 & 14.07 & 23.42 & 58 & 66 & 163 \\
\hline
\end{tabular}

The fuzzy robustness index is directly related to the change in fuzzy compatibility value. The higher the change in compatibility value, the lower the value of fuzzy robustness index and vice-versa. The higher the value of the fuzzy robustness index, the higher the systems ability to adapt to changing conditions. Fuzzy flood robustness index of the Medway Creek is expressed using a color ramp, with blue representing location with low robustness and dark brown with high robustness (Fig. 6).

When the acceptable level of flood damage changes from a conservative level to a neutral level, the fuzzy flood robustness index value is in the range from 2.94 (less robust) to 10 (highly robust). Areas near the river and the confluence on the map in Fig. (6a) are shown in blue (fuzzy flood robustness index value between 2.94 and 4.25). Land close to Medway Creek and the confluence shows lower robustness compared to the areas further away from the river which are shown in yellow (index range from 4.25 to 6.3 ), orange (index range from 6.3 to 7.10 ), green (index range from 7.11 to 9.3) or brown (index range from 9.4 to 10 ).

Adaptation to change in the acceptable level of flood damage from conservative to risky is more difficult and the value of fuzzy flood robustness index decreases. Map in Fig. (6b) shows areas near the river and the confluence with much lower value of the fuzzy flood robustness index, from 1.39 to 3 .

When the acceptable level of flood damage changes from neutral to risky, the fuzzy flood robustness index shows lower decline compared to the change from conservative to risky level of the acceptable flood damage. In the case of change from conservative to neutral level, approximately $7.97 \mathrm{~km}^{2}$ of the study region shows robustness index between 0 and 3 . For change from neutral to risky and conservative to risky, the area increases to $23.3 \mathrm{~km}^{2}$ and $32.4 \mathrm{~km}^{2}$, respectively. The more drastic change in the acceptable level of flood damage results in lower robustness. Also, locations further away from the river and low lying areas show higher robustness to flooding.

The time necessary to recover from flood depends on the severity of the flood event. The longer recovery time indicates lower resiliency. Similarly, systems that recover quickly have high level of resiliency. Fuzzy flood resiliency index map of the Medway Creek is shown in Fig. (7). The value of fuzzy flood resiliency index for the study region is expressed using a color ramp. The dark blue color represents low resiliency value of 0 - low ability for a quick recovery. Dark brown color represents the highest value of resiliency in the region (1.97) - high ability for a quick recovery.

Areas close to the Medway Creek and the confluence region are areas of low resiliency (shown in blue). The fuzzy flood resiliency index for these areas ranges from 0 (dark blue) to 0.23 (light blue). Due to low elevation just north and south of the confluence, submerged areas need more time to recover. The residential area near the confluence is subject to high flood damage that requires long recovery time - therefore low fuzzy flood resiliency index value. Regions outside the floodplain show higher resiliency. They are represented on the map in yellow (fuzzy flood resiliency index value from 0.24 to 0.34 ), orange (fuzzy flood resiliency index value from 0.35 to 0.45 ), green (fuzzy flood resiliency index value from 0.46 to 0.6 ) and brown (fuzzy flood resiliency index value from 0.61 to 1.97 ). The agricultural land north of the City of London shows high resiliency (indicated in green and brown on the map in Fig. (7)).

\section{CONCLUSIONS}

Research discussed in this paper is focusing on one possible methodology for the reliability analysis of water resources systems that is capable of: (a) addressing water resources uncertainty caused by variability and ambiguity; (b) integrating objective and subjective risk; and (c) assisting the 
water resources management based on better understanding of spatial variability of risk.

Use of fuzzy reliability analysis provides for addressing water resources management uncertainty caused by variability and ambiguity. Risk is described using a combined fuzzy reliability and vulnerability, fuzzy robustness and fuzzy resiliency. Innovative risk definition required for the application of fuzzy reliability analysis integrates objective and subjective aspects of water resources management risk. Fuzzy reliability analysis has been successfully extended into a spatial fuzzy reliability analysis for taking explicitly into consideration spatial variability of water resources management risk.

Fuzzy reliability indices implemented through the GIS are used to illustrate the proposed methodology for the flood reliability analysis of Medway Creek within the City of London. The study region is of high significance as the population of the city grows and development in the floodplains may become a major source of future risk to residents and economy of the community. The study region consists of residential and agricultural land. A floodplain map is generated first for the study region and then the damage is estimated based on the depth-damage relationships. This work examines the damage that may occur to buildings, residential and agricultural land based on the 500 year flood. Fuzzy performance indices are used to assess the ability of the study area to withstand possible flooding conditions.

Ambiguity in the definition of failure is expressed by the fuzzy membership function of acceptable level of flood damage. As the acceptable level of flood damage increases, more land becomes exposed to the higher level of flood damage. Different patterns of flood damage exposure are obtained for the various types of land use.

The final results of the fuzzy flood reliability analysis are presented using maps that show the variation of reliabilityvulnerability, robustness and resiliency in space. Quantitative analysis of maps generated by the proposed methodology shows that the high acceptable level of flood damage can result in larger area of high flood reliability and high flood resiliency and the low acceptable level of flood damage can result in low flood reliability and low flood resiliency.

Maps of fuzzy reliability indices provide additional decision support for (a) land use planning, (b) selection of appropriate flood mitigation strategies, (c) planning emergency management measures, (d) selecting an appropriate construction technology for flood prone areas, and (e) flood insurance. Use of the acceptable partial flood damage concept allows for the expression of decision maker's risk preferences and the examination of their impact on flood management decisions.

\section{ACKNOWLEDGEMENTS}

Funding from the Public Safety and Emergency Preparedness (PSEP) and the National Sciences and Engineering Research Council (NSERC) of Canada to carry out this work is greatly appreciated.

\section{REFERENCES}

[1] C. W. Ling, "Characterising Uncertainty: A Taxonomy and an Analysis of Extreme Events", MSc Thesis, School of Engineering and Applied Science, University of Virginia, 1993.

[2] S. P. Simonovic, "Risk in sustainable Water Resources Management". In Sustainability of Water Resources Under Increasing Uncertainty, IAHS Publ., vol. 240, pp. 3-17, 1997.

[3] W. W. Lowrance, Of Acceptable Risk. William Kaufman, Inc., Los Altos, CA, 1976.

[4] P. Slovic, The Perception of Risk. Earthscan, London, UK, 2000.

[5] H-S. Ang, and H. Tang, Probability Concepts in Engineering Planning and Design. New York, USA: John Wiley \& Sons, Inc., 1984.

[6] S. G. Vick, Degrees of Belief: Subjective Probability and Engineering Judgment. ASCE Press, USA, 2002.

[7] I. El-Baroudy, and S. P. Simonovic, "Fuzzy criteria for the evaluation of water resource systems performance", Water Resource Research, vol. 40, (10), 2004.

[8] I. El-Baroudy, and S. P. Simonovic, "Application of the Fuzzy Performance Measures to the City of London Water Supply System", Can. J. Civil Eng., vol. 33(3), pp. 255-266, 2006.

[9] S. S. Ahmad, and S. P. Simonovic, "A methodology for spatial fuzzy reliability analysis", Appl. GIS J., vol. 3(1), pp. 1-42, 2007.

[10] M. Morris-Oswald, and S. P. Simonovic, Assessment of the Socia Impacts of Flooding for use in Flood Management in the Red River Basin. Report prepared for the International Joint Commission, Slobodan P. Simonovic Consulting Engineer Inc., Winnipeg (available from Slobodan P. SIMONOVIĆ Consulting Ltd., 10 Pitcarnie Cr., London, Ontario, N6G 4N4, Canada), 1997.

[11] S. P. Simonovic, "Social Criteria for Evaluation of Flood Control Measures - Winnipeg Case Study", Urban Water (special issue 'Non-Structural Measures in Urban Flood Control' edt. B. Braga), vol.1, No.2, pp. 167-175, 1999.

[12] Nirupama, and S. P. Simonovic. Role of Remote Sensing in Disaster Management. Technical Report, Institute of Catastrophic Loss Reduction, London, Ontario, Canada(available from the web http://www.iclr.org/research/publications_floods.htm last accessed August 2006), 2002.

[13] Ontario Ministry of Natural Resources, Flood Damage Estimation Guide. Queen's Printer for Ontario, Canada, 1990. 